\title{
From subdiffusion to superdiffusion of particles on solid surfaces
}

\author{
A. M. Lacasta, ${ }^{1}$ J. M. Sancho, ${ }^{2}$ A. H. Romero, ${ }^{3}$ I. M. Sokolov, ${ }^{4}$ and K. Lindenberg ${ }^{5}$ \\ ${ }^{1}$ Departament de Física Aplicada, Universitat Politècnica de Catalunya, Avinguda Doctor Marañon 44, E-08028 Barcelona, Spain \\ ${ }^{2}$ Departament d'Estructura i Constituents de la Matèria, Facultat de Física, Universitat de Barcelona, Diagonal 647, \\ E-08028 Barcelona, Spain \\ ${ }^{3}$ Advanced Materials Department, IPICyT, Apartado Postal 3-74 Tangamanga, 78231 San Luis Potosí, SLP, Mexico \\ ${ }^{4}$ Institut für Physik, Humboldt Universität zu Berlin, Newtonstrasse 15, 12489 Berlin, Germany \\ ${ }^{5}$ Department of Chemistry and Biochemistry 0340, and Institute for Nonlinear Science, University of California, \\ San Diego, La Jolla, California 92093-0340, USA \\ (Received 29 July 2004; published 15 November 2004)
}

\begin{abstract}
We present a numerical and partially analytical study of classical particles obeying a Langevin equation that describes diffusion on a surface modeled by a two-dimensional potential. The potential may be either periodic or random. Depending on the potential and the damping, we observe superdiffusion, large-step diffusion, diffusion, and subdiffusion. Superdiffusive behavior is associated with low damping and is in most cases transient, albeit often long. Subdiffusive behavior is associated with highly damped particles in random potentials. In some cases subdiffusive behavior persists over our entire simulation and may be characterized as metastable. In any case, we stress that this rich variety of behaviors emerges naturally from an ordinary Langevin equation for a system described by ordinary canonical Maxwell-Boltzmann statistics.
\end{abstract}

DOI: 10.1103/PhysRevE.70.051104

PACS number(s): 05.40.-a, 68.35.Fx, 68.43.Jk

\section{INTRODUCTION}

Diffusion processes of atoms, molecules, and clusters of molecules on surfaces have been subjects of research for many decades due to their intrinsic interest and their technological importance. Some examples of modern applications include self-assembled molecular film growth, catalysis, and surface-bound nanostructures [1,2]. Also, many techniques that are used to characterize the growth of surfaces are based on the diffusion and subsequent adsorption of particles or molecules. These applications have led to a recent resurgence of interest in such processes, but now involving the motion of small and large organic molecules [3,4] and of adsorbed metal clusters composed of tens and even hundreds of atoms $[5,6]$.

Recent research activity has been both experimental and theoretical [1-12], the principal questions in these studies being the determination of the jump lengths of large molecules or clusters on surfaces, and the diffusion asymmetry along some surface crystallographic directions or along particular paths when there are many obstacles such as steps and/or impurities to negotiate. One of the more exciting experiments has involved the direct observation of the surface mobility of fairly large organic molecules using a newly devised method of independently measuring the mean square displacement and the hopping rate of these molecules, and then taking advantage of a well known relation between them to extract the root mean square jump lengths [4]. These experiments seem to indicate that, as with single atoms in some cases $[13,14]$, long jumps spanning multiple lattice spacings may play a dominant role in the diffusion of these molecules. Another "experiment," this one numerical (molecular dynamics), has led to the prediction that clusters of hundreds of $\mathrm{Au}$ atoms will exhibit Lévy-type power-law flight length and sticking time distributions [6]. On the other hand, the theoretical literature on the subject of surface dif- fusion tends to be rather complex and, most importantly, tends to invoke Lévy walks or flights [15] as a model input. While these approaches can provide insights on the effects of other elements of the model on the dynamics of particles on surfaces, they provide little insight on how the motion involving long jumps comes about in the first place. These simulations, which aim to reproduce realistic systems, do not stress the generic, minimal ingredients of such behavior.

While a microscopically accurate analysis of surface diffusion requires extensive calculations (e.g., ab initio or molecular dynamics), even the most powerful currently available computers cannot carry such calculations to anywhere near experimentally relevant time scales $[14,16]$. Moreover, current experimental probes of the topography of surfaces, scanning tunneling microscopy and atomic force microscopy, are usually carried out at relatively high temperatures, which leads to additional difficulties for first-principles calculations. Therefore, simpler approaches are essential and valuable [17]. The scenario developed in this paper is of the simple Langevin type. It models classical particles moving in a two-dimensional potential, periodic or random, under the action of $\delta$-correlated Gaussian thermal fluctuations and the associated linear dissipation, the important control parameter of the model being the friction coefficient. In our search for different behaviors as a function of the control parameter, we must be mindful of the fact that experimental observations are not necessarily strictly asymptotic, that is, that a particular behavior may be transient. Transient behaviors may of course persist for very long times, but to find them theoretically it is not enough to carry out an asymptotic analysis. We find that, in spite of the simplicity of the model, it is able to reproduce the entire range of experimentally and computationally observed phenomenologies, ranging from superdiffusion through large-step diffusion all the way to subdiffusion. In particular, we show that Lévy-like statistics appear quite naturally within the usual Langevin scheme for under- 
damped motion in a periodic potential. It is important to stress that in this case the Lévy-behavior emerges as an intermediate asymptotic for an ensemble of particles with a Maxwell-Boltzmann distribution of velocities.

Our paper is organized as follows. In Sec. II we present the Langevin model and briefly list the quantities to be considered in subsequent sections to characterize the motion of an ensemble of particles on a surface. In Sec. III numerical results are presented for the periodic surface potential, as are analytic results for the dependence of the diffusion coefficient on friction obtained in detail in the Appendix. Section IV shows our results for the random surface potentials. Finally, we conclude with a summary and some future directions in Sec. V.

\section{THE MODEL}

We study the diffusion of a particle in a two-dimensional potential $V(x, y)$ of characteristic length scale $\lambda$, in the presence of thermal noise and the associated dissipation. The model is embodied in the equations of motion,

$$
\begin{aligned}
& m \ddot{x}=-\frac{\partial}{\partial x} V\left(\frac{x}{\lambda}, \frac{y}{\lambda}\right)-\mu \dot{x}+\xi_{x}(t), \\
& m \ddot{y}=-\frac{\partial}{\partial y} V\left(\frac{x}{\lambda}, \frac{y}{\lambda}\right)-\mu \dot{y}+\xi_{y}(t),
\end{aligned}
$$

where $m$ is the mass of the particle and an overdot denotes a derivative with respect to $t$. The generalization to distinct length scales $\lambda_{x}$ and $\lambda_{y}$ is straightforward. The parameter $\mu$ is the coefficient of friction, and the $\xi_{i}(t)$ are mutually uncorrelated white noises that obey the fluctuation-dissipation relation

$$
\left\langle\xi_{i}(t) \xi_{j}\left(t^{\prime}\right)\right\rangle=2 \mu k_{B} T \delta_{i j} \delta\left(t-t^{\prime}\right) .
$$

Equations (1) can be written in terms of the scaled dimensionless variables

$$
r_{x}=\frac{x}{\lambda}, \quad r_{y}=\frac{y}{\lambda}, \quad \tau=\sqrt{\frac{V_{0}}{m}} \frac{t}{\lambda},
$$

where $V_{0}$ is some measure (e.g., the maximum or the mean maximum) of the potential. In terms of these variables, and with an overdot now denoting a derivative with respect to $\tau$,

$$
\begin{aligned}
& \ddot{r}_{x}=-\frac{\partial}{\partial r_{x}} \mathcal{V}\left(r_{x}, r_{y}\right)-\gamma \dot{r}_{x}+\zeta_{x}(\tau), \\
& \ddot{r}_{y}=-\frac{\partial}{\partial r_{y}} \mathcal{V}\left(r_{x}, r_{y}\right)-\gamma \dot{r}_{y}+\zeta_{y}(\tau),
\end{aligned}
$$

where $\mathcal{V}\left(r_{x}, r_{y}\right)=V(x / \lambda, y / \lambda) / V_{0}$ is the dimensionless potential, and the scaled noise obeys the fluctuation-dissipation relation

$$
\left\langle\zeta_{i}(\tau) \zeta_{j}\left(\tau^{\prime}\right)\right\rangle=2 \gamma \mathcal{T} \delta_{i j} \delta\left(\tau-\tau^{\prime}\right)
$$

This scaling serves to stress that there are only two independent parameters in this model, the scaled temperature $\mathcal{T}$ and the scaled dissipation $\gamma$ :

$$
\mathcal{T}=k_{B} T / V_{0}, \quad \gamma=\mu \lambda / \sqrt{m V_{0}} .
$$

The scaled temperature will be fixed at a value smaller than unity so that the thermal fluctuations do not overwhelm the potential, but not too much smaller than unity so that activated passage over potential barriers is possible within a reasonable time.

The model (1) is a "standard" Brownian motion model. It relies on well-established ideas of statistical mechanics and invokes nothing special about the fluctuations and the dissipation. The random forces are normal thermal fluctuations, Gaussian and $\delta$ correlated, and the dissipative forces that accompany the fluctuations are constructed so as to ensure thermal equilibration. Nevertheless, we argue that many of the dynamical features of a particle evolving under these equations of motion have not been investigated until recently [18]. Furthermore, we assert that it is not necessary to inject special assumptions such as Lévy flights or special memory effects into models of surface diffusion, but that these features appear naturally from this standard model, thus placing the entire range of phenomena observed in surface diffusion on an equal common footing. Furthermore, since recent experimental and theoretical results have been presented for a variety of surfaces, we explore periodic as well as random surface potentials, the latter generated according to a given distribution and with a given short-range spatial correlation.

The most straightforward quantity to characterize the surface diffusion process is the familiar mean square displacement of the particle,

$$
\left\langle r^{2}(\tau)\right\rangle=\left\langle\left[r_{x}(\tau)-r_{x}(0)\right]^{2}\right\rangle+\left\langle\left[r_{y}(\tau)-r_{y}(0)\right]^{2}\right\rangle .
$$

Normal diffusive behavior is characterized by a linear dependence on time, $\left\langle r^{2}(\tau)\right\rangle \sim D \tau$, where $D$ is the diffusion coefficient, while nondiffusive behavior shows a different time dependence, $\left\langle r^{2}(\tau)\right\rangle \sim \tau^{\alpha}$, with $\alpha<1$ for subdiffusive behavior and $\alpha>1$ for superdiffusive motion. Not only do we seek to establish the nature of the motion as a function of the friction coefficient as measured by moments such as the mean square displacement, but we seek to establish a theoretical framework that will allow us to fully understand and predict the nature of the motion under realistic circumstances that may include more complex geometries. Toward this purpose we will explore the dependence of the diffusion coefficient $D$ on friction at large time scales.

Another characterization of the process at intermediate time scales is obtained from the probability density function (PDF) $P(r, \tau)$ of particle displacements $r$ at time $\tau$. In particular, this PDF reflects the long stretches of ballistic motion evident in the intermediate time dynamics in the low friction regime. Indeed, long ballistic excursions lead to a specific behavior of this distribution that in turn implies that the velocity of these particles remains correlated over considerable time intervals. The best way to characterize such correlations is in terms of the velocity power spectrum $S(\omega)$ $=\langle\mathbf{v}(\omega) \mathbf{v}(-\omega)\rangle$. We note that this spectrum is directly connected with the asymptotic diffusion coefficient of the particle. According to the Taylor-Kubo formula, $D=\int_{0}^{\infty} C(\tau) d \tau$, where $C(\tau)=\left\langle\mathbf{v}\left(\tau^{\prime}\right) \mathbf{v}\left(\tau^{\prime}+\tau\right)\right\rangle$ is the velocity-velocity correlation function. The latter is connected with $S(\omega)$ by the 


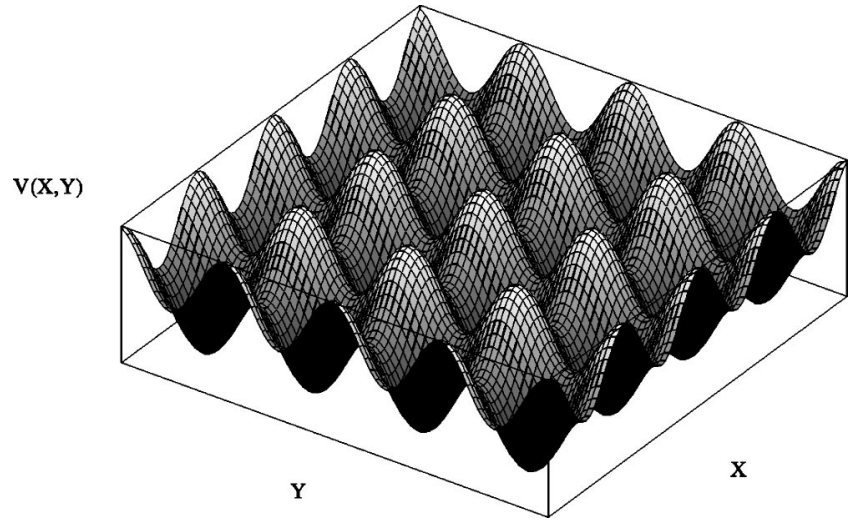

FIG. 1. A finite portion of the much larger two-dimensional periodic potential in which a particle diffuses.

Wiener-Khinchin relation, $S(\omega)=\int C(\tau) e^{i \omega \tau} d \tau$. These various observables are studied below for both periodic and random surface potentials.

\section{PERIODIC POTENTIAL}

We consider the periodic potential

$$
\begin{aligned}
V(x, y) & =V_{0} \cos \left(\frac{\pi x}{\lambda}+\frac{\pi y}{\lambda}\right) \cos \left(\frac{\pi x}{\lambda}-\frac{\pi y}{\lambda}\right) \\
& =\frac{V_{0}}{2}\left[\cos \left(\frac{2 \pi x}{\lambda}\right)+\cos \left(\frac{2 \pi y}{\lambda}\right)\right],
\end{aligned}
$$

which has maxima at positions $(n \lambda, m \lambda)$ and minima at $((n$ $\left.\left.+\frac{1}{2}\right) \lambda,\left(m+\frac{1}{2}\right) \lambda\right)$, where $n$ and $m$ are integers. The barrier height at the saddle points is $V_{0}$. This potential is shown in Fig. 1. In Fig. 2 we show examples of trajectories obtained for two different friction coefficients upon numerical simulation of the equations of motion. In our simulation we have used $\lambda=4$ and $m=1$, with a fixed scaled temperature $\mathcal{T}=0.2$ and the scaled dissipation as the independent parameter. Note that since the potential can be generated analytically and the equations of motion are continuous, the system is infinite and it is not necessary to specify boundary conditions. One of the trajectories [panel (a)] is for large friction, and the particle is seen to follow what appears to be typical diffusive motion characterized by short steps of length $\lambda$ along the crystallo-
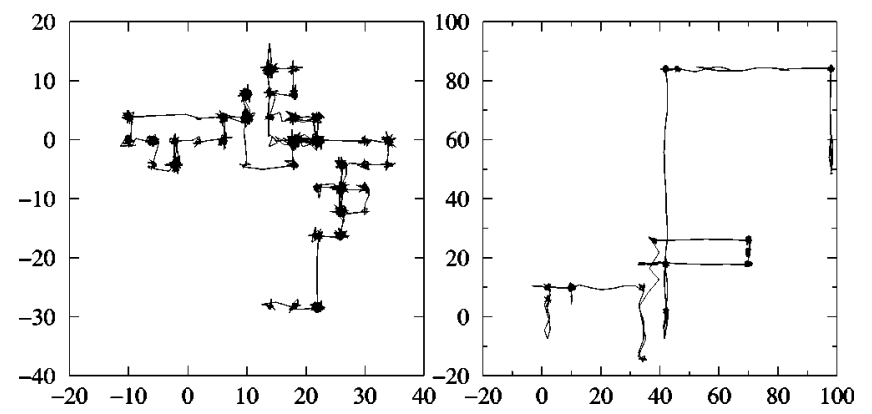

FIG. 2. Left: A trajectory for $\gamma=1$ over $t=20000$ time units. Right: A trajectory for $\gamma=0.04$ over $t=15000$ time units. The period of the potential is $\lambda=4$. Note the different scales in the two panels.

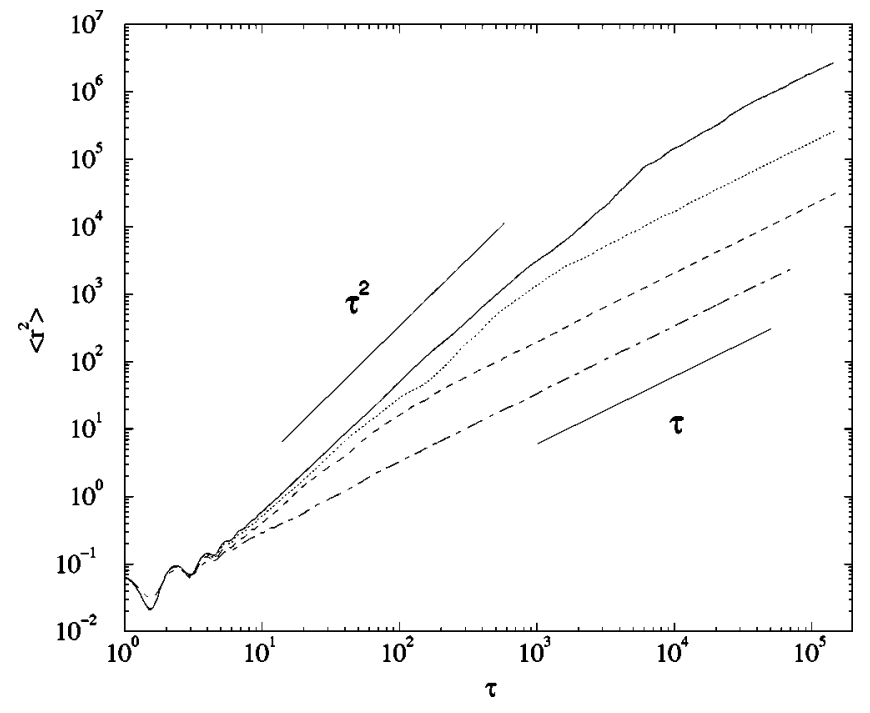

FIG. 3. Mean square displacement vs time for an ensemble of 5000 particles in the periodic potential with a Maxwell-Boltzmann initial distribution. $\gamma=0.0004$ (solid line), 0.004 (dotted line), 0.04 (dashed line), and 0.4 (dot-dashed line).

graphic directions. The other trajectory [panel (b)] corresponds to small friction and clearly shows the preponderance of long $(\gg \lambda)$ tracks along one crystallographic direction before turning to another.

The evolution of the mean square displacement $\left\langle r^{2}\right\rangle$, averaged over a set of 5000 particles, is shown in Figs. 3 and 4 for several friction coefficients and different initial conditions. In Fig. 3 the particles are initially deposited in a square of side $2 \pi$ around the center of the system according to a Boltzmann equilibrium distribution for the positions and for the velocities, while in Fig. 4 they are initially located at the center of the system with zero initial velocity. Differences in the two cases are observed at short times. At very long times

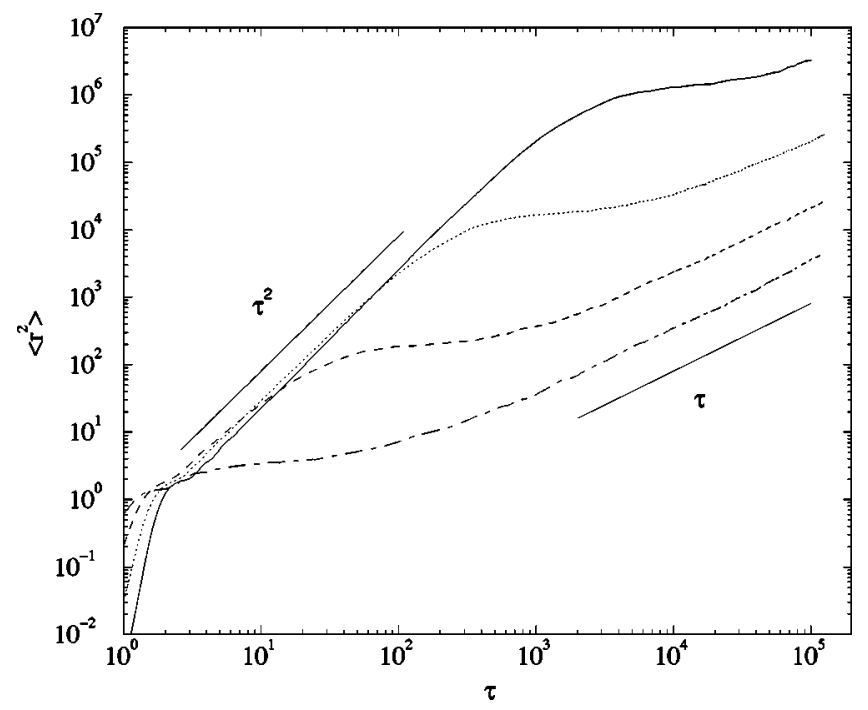

FIG. 4. Mean square displacement vs time for an ensemble of 5000 particles in the periodic potential with zero velocity, and located at the origin. $\gamma=0.0004$ (solid line), 0.004 (dotted line), 0.04 (dashed line), and 0.4 (dot-dashed line). 


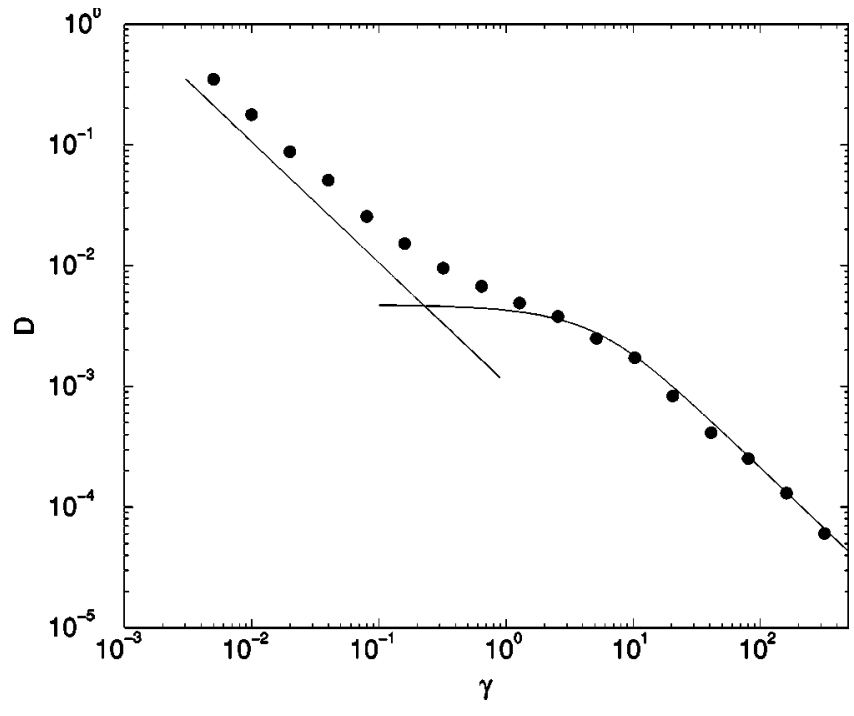

FIG. 5. Diffusion coefficient as a function of $\gamma$ for the periodic potential.

the memory of the initial condition is lost, and in all cases the motion is diffusive, as expected. Regardless of initial condition, for small $\gamma$ and at intermediate times there is clear superdiffusive ballistic $(\alpha=2)$ behavior over several decades of time, reflective of the long straight stretches seen in the low- $\gamma$ trajectory in Fig. 1. The ballistic behavior is observed over a time range of $O\left(\gamma^{-1}\right)$, and one might be tempted to conclude that it is therefore a trivial generalization of the motion of a free damped particle. However, the situation here is quite different. The motion is of an ensemble of particles whose initial energy distribution is either the Boltzmann distribution appropriate to the temperature $\mathcal{T}$ (Fig. 3), or all of which initially have zero velocity (Fig. 4), and the mean square displacement is an average over all the particles. The particles with energies lower than the barrier height, which includes most of the particles, are at first trapped in the original potential well. Others (those with a higher initial energy, and those that get kicked up sufficiently in energy by the thermal fluctuations) move out of one well only to be quickly trapped again, perhaps into a neighboring well. A few particles, those that either start with or acquire the highest velocities according to the Maxwell-Boltzmann distribution, escape the initial well and move over longer distances even as they slow down through friction, until they are once again trapped. That this complex ensemble behavior should give rise to a mean square displacement that behaves as shown in Fig. 3 is not self-evident, and in fact can only be understood in more detail if we analyze the distribution of particle displacements as a function of time. We do so later in this section.

As noted above, for very long times the motion is diffusive in all cases. The diffusion coefficient $D$, evaluated numerically at long time as

$$
D=\lim _{\tau \rightarrow \infty} \frac{\left\langle r^{2}(\tau)\right\rangle}{4 \tau},
$$

is shown in Fig. 5 as a function of the friction $\gamma$. The solid
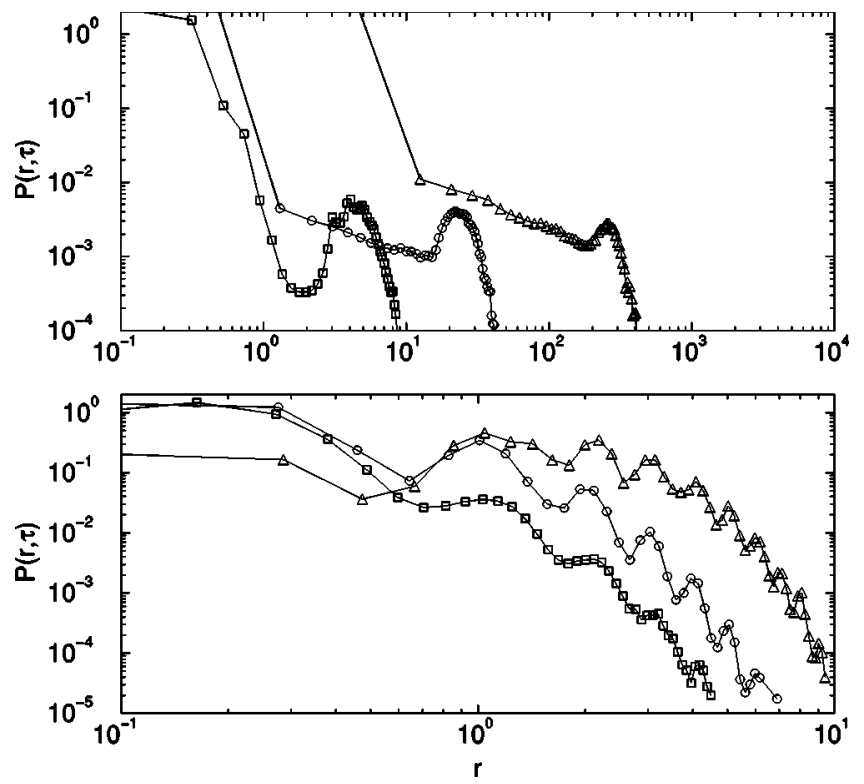

FIG. 6. Log-log plot of $P(r, \tau)$ for $\gamma=0.0004$ (top) and for $\gamma$ $=1$ (bottom) and three different values of time intervals: $\tau=20$ (squares), 100 (open circles), 1000 (triangles). Note the strong difference in scales.

lines correspond to theoretical predictions evaluated in the Appendix. There we show that in the overdamped regime [large $\gamma$; see Eq. (A6)]

$$
D \sim \frac{\pi}{\gamma} \exp \left(-\frac{1}{\mathcal{T}}\right) .
$$

In the opposite limit [small $\gamma$; see Eq. (A20)], we have

$$
D \sim \frac{\pi \mathcal{T}}{4 \gamma} \exp \left(-\frac{1}{\mathcal{T}}\right) .
$$

Both predictions fit our numerical results rather well. Note that there are no free parameters in these results. Note also that while $D \sim \gamma^{-1}$ at both high and low friction, the physical reasons are quite different. As seen in the Appendix, at high friction the mean square distance traveled between trapping events is unity independently of $\gamma$, and the mean time to escape a well and be trapped in another is proportional to $\gamma$. At low friction the mean square distance is proportional to $\gamma^{-2}$ and the mean time goes as $\gamma^{-1}$. The ratio of the two is therefore in any case proportional to $\gamma^{-1}$.

The diffusion coefficient characterizes only the very longtime asymptotic dynamics. To characterize the process at intermediate time scales we introduce the probability distribution function $P(r, \tau)$ of particle displacements $r$ at time $\tau$. In particular, this PDF reflects the long stretches of ballistic motion evident in the intermediate time dynamics in the lowfriction regime. The PDF is shown in Fig. 6 (top) for $\gamma$ $=0.0004$ and three different time intervals $\tau$ for an initially equilibrated ensemble. For comparison, we also show typical PDF's for high damping ( $\gamma=1$, bottom) for the three time intervals. In the high- $\gamma$ curves the highest maximum corresponds to no jumps (by far the most likely event at short times). The next is associated with jumps to a nearest neigh- 
bor well, and so on. In contrast, the low- $\gamma$ curves show a very different behavior, with features strongly resembling those of a Lévy-walk model $[19,20]$ : a peak at small displacements, a power-law intermediate regime, and a side hump at high displacements. Each of these is a distinct signature of Lévy-walk-like dynamics, but one must be cautious in the detailed interpretation of these components, because in our results they are not attributable to exactly the same sources as in the Lévy walk, nor do the exponents fit the Lévy walk scheme directly. In our system the persistent small-displacement peak is associated with long trapping periods during which a particle does not move at all because its energy is not sufficient to overcome the barrier. The high displacement peak, which moves outward with velocity of order unity, is associated with ballistic motion of those particles that acquire enough energy to move (and lose it very slowly). Genuine Lévy-walk dynamics also exhibit a low displacement peak and a superdiffusive peak. In the Lévy walk, the persistent small-displacement peak stems from the distribution of excursion lengths, whereas the highdisplacement peak is associated with laminar events in which a particle started moving before the observations started and are still moving without interruption at time $t$.

These small- and large-displacement domains are separated by a power-law behavior. However, here some important differences must also be stressed. In the Lévy walk there is a particular relation between the exponent $\alpha$ of the time in the mean square displacement $\left\langle r^{2}\right\rangle \sim t^{\alpha}$ and the slope $\mu$ of the power-law regime of the distribution, $P(r, \tau) \sim \tau / r^{\mu}$, namely, $\alpha=4-\mu$. Furthermore, the distribution with the features that we are describing is observed in the regime $2<\mu<3$, that is, $1<\alpha<2$. In our distribution the intermediate power-law regime reflects ballistic transport, and yet the slope in our power-law regime is smaller than unity (approximately 0.7 ). Nevertheless, the qualitative features of our distribution track those of the Lévy walk. Our side hump is strongly broadened whereas the side hump in the Lévy-walk model is associated with motion at a single constant velocity. In our case the velocity varies according to the equilibrium MaxwellBoltzmann distribution. Note that the existence of the pronounced side hump moving with the velocity of the order of unity reflects the fact that the particles performing long steps ("flights") are those in the tail of the Maxwellian velocity distribution. This contribution to the PDF in a potential system arises from a small subset of particles and is thus to be distinguished from that of a typical underdamped free Brownian particle [21]. In summary, in our underdamped system the slower particles are trapped and localized around a potential minimum. They do not contribute to transport and lead to the first peak in the PDF $P(r, \tau)$. Particles with higher energies begin moving essentially ballistically, thus contributing to the side hump of the PDF that moves toward the right with increasing times in the first panel in Fig. 6. These particles eventually become trapped due to friction, and these trapping events lead to the power-law portion of the PDF. Fewer and fewer particles (only those in the ever smaller highest-velocity tails of the distribution) continue moving ballistically. The side hump thus becomes narrower and lower with time. At long times, $\tau \gg 1 / \gamma$, both the longtrapping and ballistic features are of course no longer present as diffusive motion dominates the behavior.

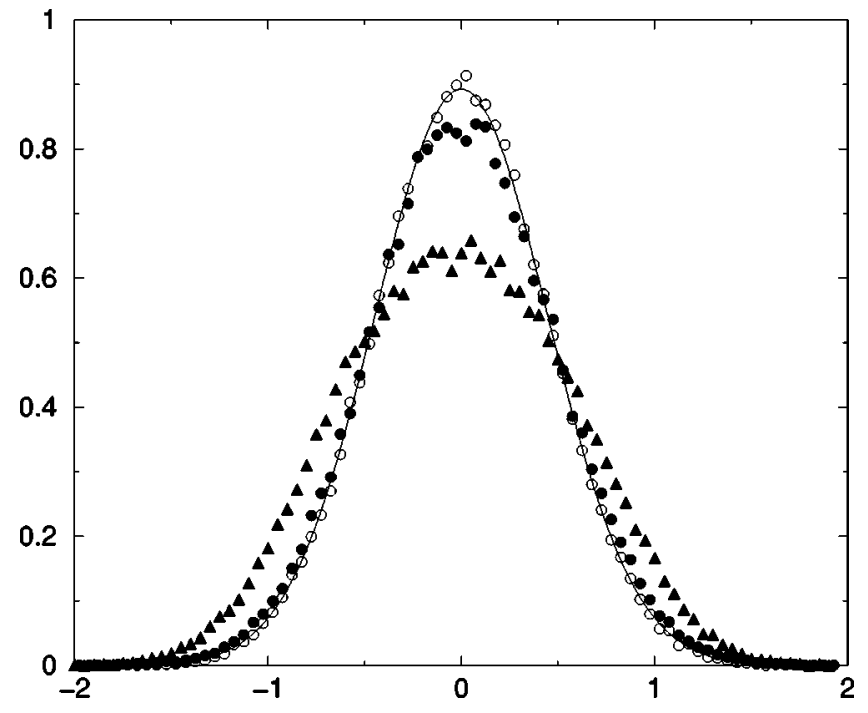

FIG. 7. Probability distribution of the velocity for two different initial conditions compared with a Maxwell distribution (solid curve) for the same temperature. Triangles: distribution at $\tau=50$ for zero velocity initial condition. Circles: distributions at $\tau=100$ for zero velocity (solid circles) and Maxwell (open circles) initial distributions $(\gamma=0.04)$.

We emphasize that the behavior described above is universal and is not strongly dependent on whether the initial conditions are equilibrium or zero-velocity ones (although the exact heights of the peaks do). In Fig. 7 the velocity probability distributions of our system at finite times are compared to a Maxwell distribution. When we implement Maxwell-Boltzmann initial conditions, the overall velocity distribution obtained in our simulations by time sampling is purely Maxwellian at all times. For the nonequilibrium initial conditions where we start with zero velocity at the saddle point of the potential, some nonequilibrium transient phenomena are observed in the small deviations from the Maxwell form. We also note that the velocity distribution thermalizes at times of the order of $10 \gamma^{-1}$. The times in Fig. 7 correspond to $t=2 \gamma^{-1}$ and $4 \gamma^{-1}$.

The long, ballistic excursions that lead to the power-law and side hump contributions to the particle displacement distribution function imply that the velocities of these particles remain correlated over considerable time intervals. This aspect is most directly characterized by a study of the velocity power spectrum $S(\omega)=\langle\mathbf{v}(\omega) \mathbf{v}(-\omega)\rangle$. In the case of diffusive motion one can extract the diffusion coefficients as

$$
D=\frac{1}{4}\left[S_{x x}(\omega=0)+S_{y y}(\omega=0)\right],
$$

where $S_{i i}(\omega)$ is the power spectrum of the Cartesian component $i$ of the velocity vector. The behavior of $S(\omega)$ for different values of the friction coefficient $\gamma$ is shown in Fig. 8. The structure of the power spectrum mirrors the underlying dynamics. Thus, an evident feature of $S(\omega)$ is the existence of pronounced peaks at the frequency $\omega_{0}=\pi \sqrt{2}$ of small oscillations in one well performed by particles trapped in a well. At low frequencies, $\omega_{0} \ll 1$, a power-law growth of $S(\omega)$ is 


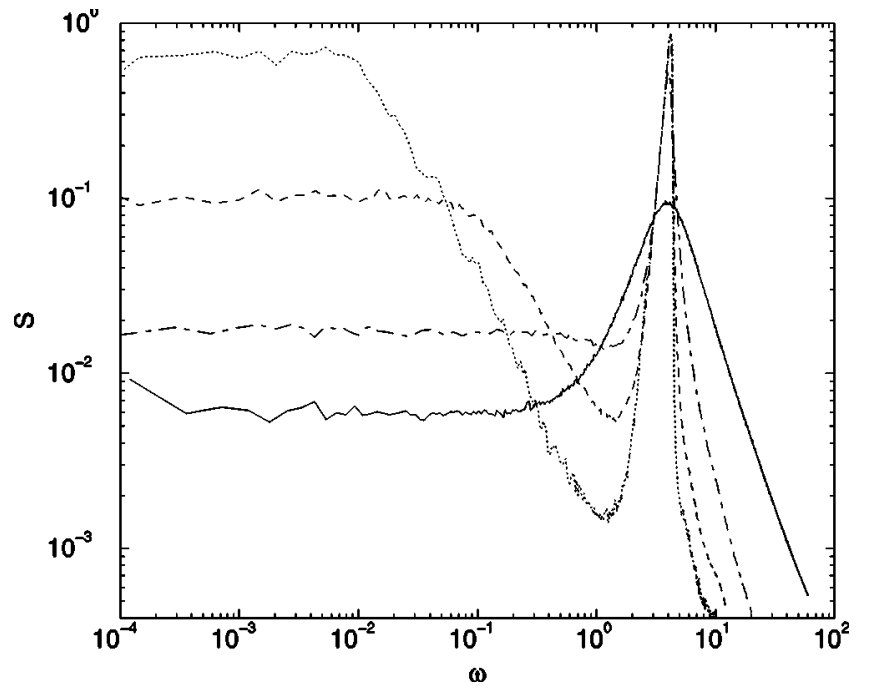

FIG. 8. $S(\omega)$ for $\gamma=0.004,0.04,0.4$, and 4 in decreasing order.

observed. This is reflective of the persistent time correlations associated with ballistic excursions. The slope $\sim 1$ of this growth corresponds to $C(\tau) \approx$ const and to the ballistic growth of the mean square displacement. At even smaller frequencies the power spectrum crosses over to $S(\omega)$ $=$ const $=4 D$, indicating full decorrelation and emergence of pure diffusion.

\section{RANDOM POTENTIAL}

Surfaces are usually not completely crystalline or regular because of the presence of vacancies, defects, and other types of disorder. One type of surface disorder is represented by a random potential with spatial correlations that can model the presence of some spatial finite-ranged order. An algorithm that can be used to generate surfaces with any given spatial correlation has been presented in Refs. [22-24]. We have implemented this algorithm for a random potential surface with a Gaussian distribution and exponential correlation function. In terms of the vectors $\mathbf{x}=(x, y)$ and $\mathbf{x}^{\prime}$ $=\left(x^{\prime}, y^{\prime}\right)$, our potential surface has the correlation property

$$
\left\langle V(\mathbf{x}) V\left(\mathbf{x}^{\prime}\right)\right\rangle=g\left(\mathbf{x}-\mathbf{x}^{\prime}\right),
$$

with

$$
g\left(\mathbf{x}-\mathbf{x}^{\prime}\right)=\frac{\varepsilon}{2 \pi \lambda^{\prime 2}} e^{-\left|\mathbf{x}-\mathbf{x}^{\prime}\right|^{2} / 2 \lambda^{\prime 2}}
$$

which is parametrized by the intensity $\varepsilon$ and the characteristic length $\lambda^{\prime}$ that we take to be the same as the length scale in the periodic potential $\left(\lambda^{\prime}=4\right)$. Note that the average potential height is a combination of the parameters. A typical surface generated with this algorithm and of average potential height $V_{0}$ equal to that of the periodic potential is shown in Fig. 9. In our particular case, we have done our simulations on a square grid of size $L=4096$ with $\delta x=1.0$. Even though our potential is generated on a grid, extrapolation of forces can be used to calculate the force at any point, which

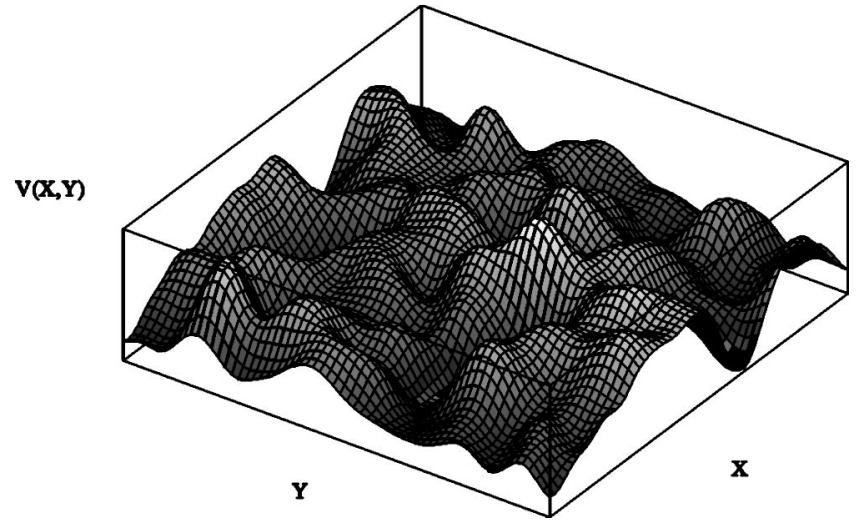

FIG. 9. Random potential with the same average potential height as in the periodic case.

is a necessary step in the dynamical simulation. We have used the parameter $\varepsilon=100$. In this case we must generate finite systems. Rather than impose boundary conditions, we stop each simulation sequence whenever the first particle reaches a boundary.

Typical trajectories associated with this potential at high and low friction are shown in Fig. 10. Again, the trajectories are entirely different, that associated with high friction consisting of extremely short steps followed by random changes in direction while that associated with low friction again shows a pronounced directional persistence.

An analysis of the exponents in the mean square displacement formula shows the entire range of behaviors from subdiffusive to superdiffusive with changing friction. In Fig. 11 we show $\left\langle r^{2}(\tau)\right\rangle$, averaged over 5000 particles, as a function of time for several values of $\gamma$. A detailed analysis of these trajectories presents a rich phenomenology of possible different regimes. In the overdamped regime we observe clearly subdiffusive behavior $(\alpha<1)$, already known from overdamped simulations presented earlier [24]. An interesting outcome is the superdiffusive $(\alpha>1)$ behavior seen for small values of $\gamma$. This again is a signature of the Lévy-walk-type behavior. The exponent $\alpha$ as a function of $\gamma$ is plotted in Fig. 12. Each exponent is obtained as an average over 500 particles in each of 20 different realizations of the random potential, the error bars indicating the standard deviations over these statistics. For large $\gamma$ our simulations run for a long

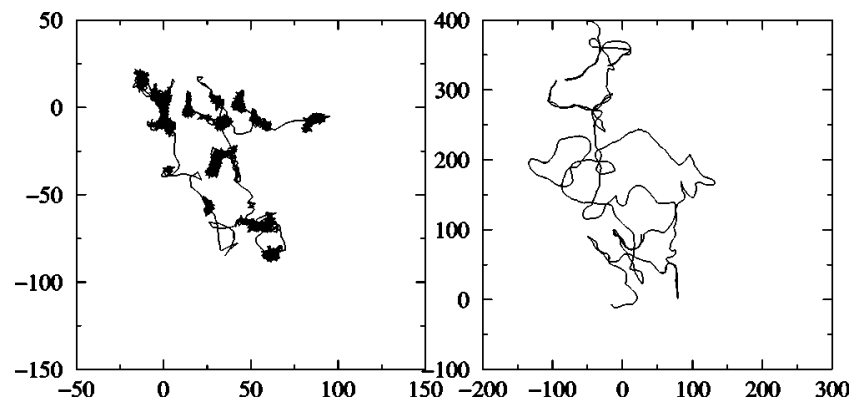

FIG. 10. Left: A trajectory for $\gamma=0.1$ over $t=250000$ time units. Right: A trajectory for $\gamma=0.0001$ over $t=2000$ time units. The characteristic length scale of the potential is $\lambda^{\prime}=4$. Note the different scales in the two panels. 


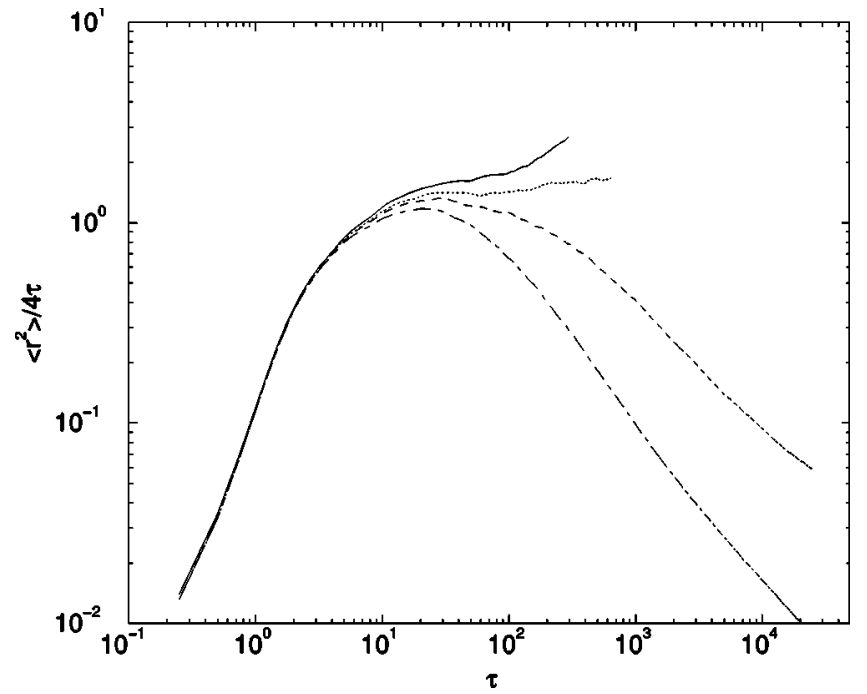

FIG. 11. Mean square displacement for a particle in the random potential with Maxwellian initial velocity distribution, for $\gamma$ $=0.0001$ (solid line), 0.001 (dotted line), 0.003 (dashed line), and 0.008 (dot-dashed line).

time and the value of $\alpha$ has been calculated on the basis of the behavior of $\left\langle r^{2}\right\rangle$ over the last time decade of the simulation for each run. In the medium- and low-damping cases the simulations do not extend over such long times (since each simulation ends when a particle reaches the system boundary). The standard deviations are larger for small $\gamma$. In part this is due to the fact that for small $\gamma$ it is more likely for a particle to reach the boundary of the system during the time of a run. Not only are the run times shorter for smaller $\gamma$, but they are also more broadly distributed as $\gamma$ decreases. In any case, the trend in the behavior of $\alpha$ as a function of the friction is clear. An unexpected result is the absence of a diffusive regime $(\alpha \approx 1)$ except in a small range of values.

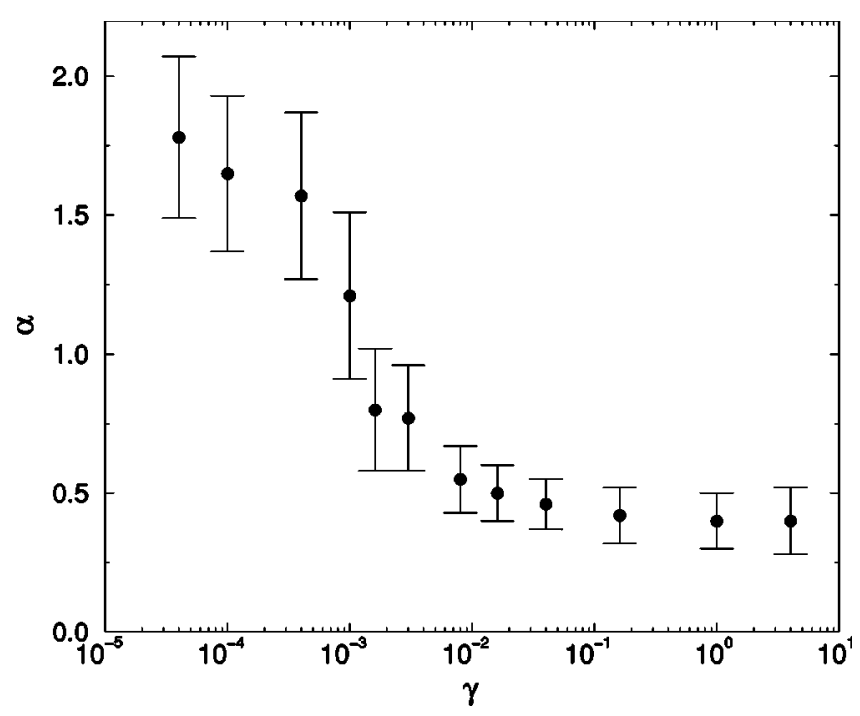

FIG. 12. Exponents $\alpha$ versus friction coefficient $\gamma$. Each point is obtained from an average over 20 realizations of the random potential and 500 particles per realization. The error bars indicate the standard deviation over these statistics.

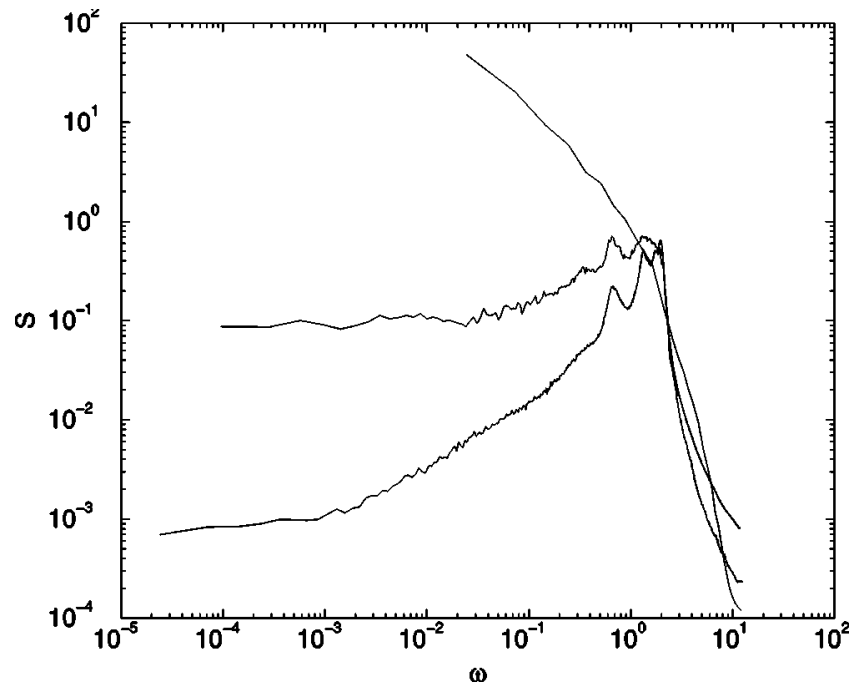

FIG. 13. $S(\omega)$ for $\gamma=0.0001,0.003$, and 0.08 (decreasing order) in the random potential with Maxwellian initial velocity distribution.

The behavior of $S(\omega)$ for the random potential is seen in Fig. 13 for several values of $\gamma$. For small $\gamma$ the spectral density grows with decreasing frequency, indicating superdiffusion, while at large $\gamma$ it decays with decreasing $\omega$, which is a signature of subdiffusion. For the intermediate value $\gamma$ $=0.003$ (close to the value at which $\alpha \approx 1$ is observed in Fig. 12) it flattens, as appropriate for diffusive behavior at long times.

In Fig. 14 the PDF $P(r, \tau)$ for the random potential is plotted. This PDF looks very different from the one for a periodic potential. Nevertheless, for the case of small friction it still exhibits some features of Lévi-walk-like dynamics.
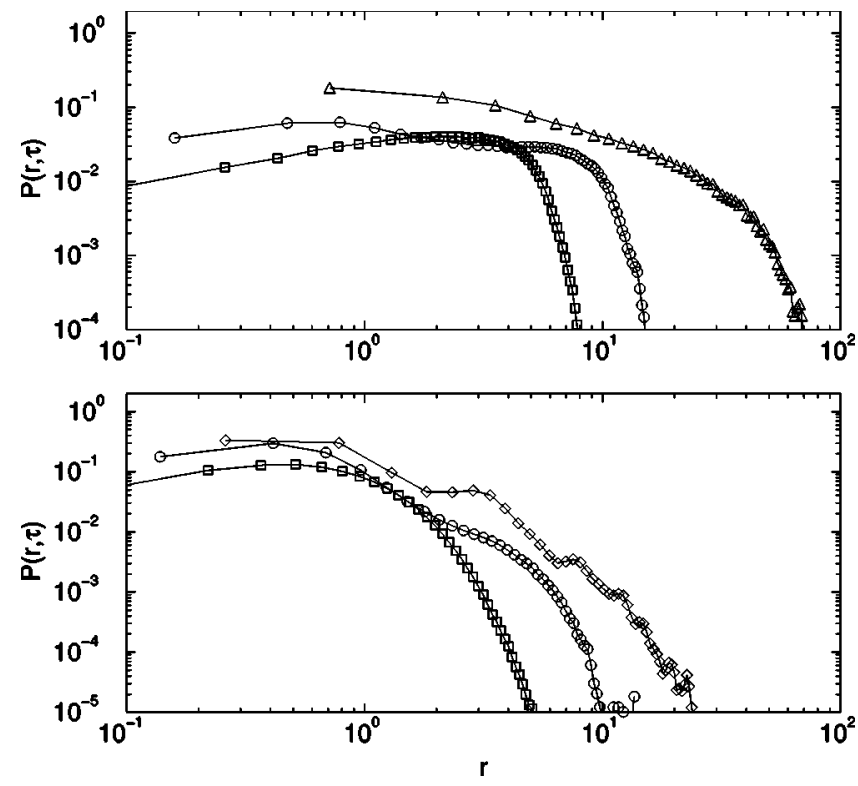

FIG. 14. Log-log plot of $P(r, \tau)$ for $\gamma=0.0001$ (top) and for $\gamma$ $=0.08$ (bottom) and various different values of time intervals: $\tau$ $=10$ (squares), 20 (open circles), 100 (triangles), and 1000 (rhombuses). 
The main difference here is the absence of the pronounced central peak due to trapping in the nearest potential well. This trapping is still evident in the high friction trajectory in Fig. 10. However, for small friction the corresponding peak in the PDF is smoothed out by the broad distribution of depths of the sampled minima. Instead, the distribution resembling a smoothed form of the genuine ballistic Lévywalk PDF appears, characterized by the central power-law dip and side peak. The side peak moves outward with a constant velocity and becomes broadened by scattering and trapping. At long times the PDF develops the tentlike central peak typical of trapping.

\section{CONCLUSIONS}

We have investigated the behavior of an ensemble of particles in a two-dimensional potential subject to thermal fluctuations described by ordinary Langevin dynamics. Our purpose has been to determine whether such an ordinary description is sufficient to produce the entire range of behaviors, from subdiffusive to superdiffusive, that has been observed in the motion of molecules on surfaces [1-12]. Indeed, we found that it is not necessary to add any further assumptions to the model to observe the full range of behaviors, at least on intermediate time scales. For a given surface potential one can introduce scaled parameters such that the only control parameters in a periodic potential are the temperature (which we have held fixed in this analysis) and the dissipation parameter $\gamma$. In the case of a random potential we introduced an additional parameter (which we also hold fixed in this analysis) to characterize the intensity of the spatial correlation function of the potential variations.

For the periodic surface potential we found that in the underdamped regime the motion of the particles includes a ballistic range that can extend over many decades of time. The probability distribution function of the displacement of the particles exhibits a structure reminiscent of that associated with Lévy walks. The PDF has a peak at short distances that arises from those particles that are trapped in their original well. The PDF also exhibits a hump that moves outward linearly with increasing time, and that is due to those particles whose energy is sufficiently high for ballistic motion over the time of observation. As these particles in turn get trapped, the amplitude and width of this hump decrease. Particles that start out moving ballistically eventually lose enough energy through damping to get trapped, and these progressive trapping events give rise to a power-law intermediate regime connecting the trapped particle peak and the ballistic particle hump. These behaviors are not observed in the case of high dissipation. The long stretches of essentially ballistic motion of the more energetic particles also make themselves apparent in the velocity power spectrum. We have ascertained that the Lévy-like features in the lowdamping case arise from a subset of energetic particles by confirming that throughout the evolution the velocity distribution of particles remains Maxwellian if the initial distribution is Maxwellian. If initially the particles have zero velocity, the distribution rather quickly settles into an essentially Maxwellian distribution. Thus, we stress again that in our model these features arise not because the average particle is subjected to any esoteric fluctuations, but rather from the fact that the energetic particles in an ordinary Maxwell distribution can move over long distances when the system is underdamped. At long times (low frequencies) the motion of the particles eventually becomes diffusive. This is most clearly seen in the behavior of the mean square displacement (which eventually grows linearly with time), and in the plateau of the velocity power spectrum at low frequencies.

While most of the results reported above are obtained numerically, we are able to calculate the diffusion coefficient $D$ that describes the long-time behavior of the system analytically for high damping (a well-known result) and also for low damping. The agreement of our analytic results for $D$ with numerical ones is good over the entire range of damping coefficients.

The case of random potential gives rise to a large variety of regimes ranging from superdiffusion to subdiffusion. While the superdiffusive behavior is probably a transient, just as in the periodic potential, subdiffusion due to multiple trapping is known to be a true asymptotic behavior when the temperature is low. However, in the case of a random potential the restrictions imposed by our numerical procedure are tighter, so that the true asymptotic behavior is difficult to reach. At short times and for weak damping the Lévy-walklike features arising from long stretches of motion of energetic particles are still evident in our simulations. For strong damping this regime is pertinent only for the shortest times and then crosses over to that typical of subdiffusive, dispersive transport.

\section{ACKNOWLEDGMENTS}

This work was supported by the MCyT (Spain) under Project No. BFM2003-07850, by the Engineering Research Program of the Office of Basic Energy Sciences at the U.S. Department of Energy under Grant No. DE-FG0386ER13606, and by a grant from the University of California Institute for México and the United States (UC MEXUS) and the Consejo Nacional de Ciencia y Tecnología de México (CoNaCyT). A.H.R. acknowledges support from Millennium Initiative, Conacyt-Mexico, under Grant No. W-8001. I.M.S. acknowledges the hospitality of the University of Barcelona under a CEPBA grant, as well as partial financial support by the Fonds der Chemischen Industrie.

\section{APPENDIX: DIFFUSION COEFFICIENT FOR HIGH AND LOW FRICTION IN A PERIODIC POTENTIAL}

We start with the assumption that a particle that has sufficient energy to move away ("escape") from a potential well will in general preferentially move along directions of lowest potential barriers, that is, along the $r_{x}$ direction at $r_{y}=1 / 2$ (or an odd multiple thereof) or along the $r_{y}$ direction with $r_{x}$ $=1 / 2$ (or an odd multiple thereof). Choosing the former, we then have the particle moving along a line in the periodic potential

$$
\mathcal{V}\left(r_{x}\right) \equiv \mathcal{V}\left(r_{x}, r_{y}=1 / 2\right)=-\sin ^{2} \pi r_{x} .
$$

Only those particles with energy $E>0$ can move. As they move they lose energy through dissipation, until they be- 
come trapped. We calculate the diffusion coefficient for the ensemble of particles according to the formula

$$
D=\left\langle l^{2}\right\rangle /(2 \tau) .
$$

Here $\left\langle l^{2}\right\rangle$ is the mean square displacement from the initial well to the well associated with the next trapping event, $\tau$ is the mean time for such a journey, and the numerical factor in the denominator is 2 instead of 4 [cf. Eq. (9)] because the motion along each stretch is one dimensional. The average is over all the particles in the ensemble, which includes those that initially have sufficient energy to move away from a potential well and also those that do not, as dictated by the Maxwell-Boltzmann distribution. For low-energy particles to be able to jump out they must acquire enough energy from the thermal fluctuations.

The mean time $\tau$ in general includes the time for a particle to acquire enough energy $(E>0)$ to move out of a well, and the time to become trapped again in a new well once it has escaped. We can calculate $\tau$ using arguments for the calculation of transition rates of the Kramers problem [25] by associating it with the inverse of a transition rate that consists of two contributions

$$
\tau=\frac{1}{2 k}=\frac{1}{2 k^{t s t} \kappa} .
$$

One contribution is the transition state theory rate,

$$
k^{t s t}=\frac{\omega_{0}}{2 \pi} e^{-1 / \mathcal{T}},
$$

where $\omega_{0}$ is the angular frequency at the well bottom, which in our dimensionless variables and for our potential is given by $\omega_{0} \equiv\left[\mathcal{V}^{\prime \prime}(1 / 2)\right]^{1 / 2}=\pi \sqrt{2}$. The other is the transmission factor $\kappa$, which depends in a nontrivial way on the damping.

Consider first the case of high friction. In the overdamped regime (large $\gamma$ ) we assume that $\left\langle l^{2}\right\rangle=1$, and the transmission factor $\kappa$ has the well-known form [25]

$$
\kappa=\frac{1}{\omega_{b}}\left[\left(\frac{\gamma^{2}}{4}+\omega_{b}^{2}\right)^{1 / 2}-\frac{\gamma}{2}\right],
$$

where $\omega_{b}^{2}=\mathcal{V}^{\prime \prime}\left(r_{b}\right)=2 \pi^{2}$ and $r_{b}$ is the position of the maximum of the potential. Thus the diffusion coefficient in this regime is

$$
D=\frac{1}{2 \pi}\left[\left(\frac{\gamma^{2}}{4}+2 \pi^{2}\right)^{1 / 2}-\frac{\gamma}{2}\right] e^{-1 / \mathcal{T}} \sim \frac{\pi}{\gamma} e^{-1 / \mathcal{T}} .
$$

Now consider the diffusion coefficient in the small- $\gamma$ limit or infradamped regime. In this regime a more elaborate analysis is required to calculate $\left\langle l^{2}\right\rangle$. The equations of motion of a particle in the potential Eq. (A1) can be expressed in the standard momentum-position form as the pair,

$$
\begin{gathered}
\dot{r}_{x}=p, \\
\dot{p}=-\gamma p-\mathcal{V}^{\prime}\left(r_{x}\right)+\zeta(\tau) .
\end{gathered}
$$

In the small- $\gamma$ limit the energy variation of a particle is slow, and it is more convenient to rewrite the dynamical equations in terms of the displacement and the energy $E=p^{2} / 2+\mathcal{V}\left(r_{x}\right)$ :

$$
\begin{gathered}
\dot{r}_{x}=\left\{2\left[E-\mathcal{V}\left(r_{x}\right)\right]\right\}^{1 / 2}, \\
\dot{E}=-2 \gamma\left[E-\mathcal{V}\left(r_{x}\right)\right]+\left\{2\left[E-\mathcal{V}\left(r_{x}\right)\right]\right\}^{1 / 2} \zeta(t) .
\end{gathered}
$$

Two quantities needed for our estimate of the diffusion coefficient can be calculated from these dynamical equations. One is the time $\tau(E)$ that it takes a particle of energy $E$ to traverse a unit distance (the scaled spatial period). The other is the energy $\Delta E$ lost by the particle when it traverses this distance [26]. To calculate $\tau(E)$ we assume that the energy $E$ remains fixed during the traversal, so that we can simply integrate Eq. (A8) for constant $E$. With the potential (A1) one obtains a standard integral:

$$
\tau(E)=\int_{0}^{1} \frac{d r_{x}}{\sqrt{2\left[E-\mathcal{V}\left(r_{x}\right)\right]}}=\frac{\sqrt{2} \lambda}{\pi \sqrt{1+E}} \mathcal{K}\left(\sqrt{\frac{1}{1+E}}\right),
$$

where $\mathcal{K}$ is the complete elliptic integral of the first kind [27]. For $E \ll 1$ the elliptic integral can be approximated by a logarithmic leading term, which leads to

$$
\tau(E) \approx \frac{1}{\pi \sqrt{2}} \ln \frac{16}{E} .
$$

Next, we estimate the energy loss that occurs during this traversal. This has been calculated in many ways, but a particularly transparent argument [26] is to neglect the fluctuations in Eq. (A9) (since particles above the barrier primarily lose energy) and integrate over a time interval $\tau(E)$. The $r_{x}$ dependence in Eq. (A9) even after this approximation still poses a problem because it leads to incomplete elliptic integrals amenable only to numerical integration. Since $r_{x}$ changes rapidly compared to $E$, it is reasonable to perform an average of the $r_{x}$-dependent term over a traversal from 0 to 1 . This leads to the approximate equation,

$$
\dot{E}=-2 \gamma F(E)
$$

where

$$
F(E) \equiv \int_{0}^{1} d r_{x} P_{E}\left(r_{x}\right)\left[E-\mathcal{V}\left(r_{x}\right)\right]
$$

with

$$
P_{E}\left(r_{x}\right)=\frac{1}{\tau(E)\left[E-\mathcal{V}\left(r_{x}\right)\right]^{1 / 2}} .
$$

The result is

$$
F(E)=\frac{\sqrt{2(1+E)}}{\pi \tau(E)} \mathcal{E}\left(\sqrt{\frac{1}{1+E}}\right),
$$

where $\mathcal{E}$ is the complete elliptic integral of the second kind [27]. Retention of terms to leading order in $E$ and $\Delta E / E$ leads to

$$
\Delta E \approx-\frac{\gamma \sqrt{8}}{\pi},
$$

which is independent of $E$. 
From the result for $\Delta E$ we can now calculate the mean square distance $\left\langle l^{2}\right\rangle$ traveled by a particle that starts with an energy above the barrier. Since the particle loses energy $\Delta E$ in each traversal of a unit distance, the distance traveled by such a particle of initial energy $E$ before being trapped is

$$
l(E) \approx-\frac{E}{\Delta E} \approx \frac{\pi E}{\gamma \sqrt{8}} .
$$

Averaging over a Boltzmann distribution of energies, we obtain,

$$
\left\langle l^{2}\right\rangle=\left(k_{B} T\right)^{-1} \int_{0}^{\infty} d E l^{2}(E) e^{-E / \mathcal{T}}=\frac{(\pi \mathcal{T})^{2}}{4 \gamma^{2}} .
$$

When a particle of positive energy $E$ moves from one potential well to the next, it does so in a time $\tau(E)$ and it loses energy $\Delta E$ in the process. If its energy is now below the barrier, it will be trapped. If not, it will keep moving, and will take a time $\tau(E-\Delta E)$ to get to the next potential well. The particle moves on until its energy is too low to continue moving. The energies have a Boltzmann distribution, so different particles will take different times to be trapped both because they are moving progressively more slowly and because they get trapped at different times in their journey. Taking these effects into account, one finally arrives at the following expression for the transmission factor $\kappa$ [26]:

$$
\kappa=\tanh \left(\frac{|\Delta E|}{2 \mathcal{T}}\right) \approx \frac{-\Delta E}{2 \mathcal{T}},
$$

where the last expression holds for very low damping. We thus finally obtain the following result for the diffusion coefficient in this regime:

$$
D \sim \frac{\pi \mathcal{T}}{4 \gamma} e^{-1 / \mathcal{T}}
$$

[1] G. E. Poirier and E. D. Plyant, Science 272, 1145 (1996); T. Yokoyama, S. Yokoyama, T. Kamikado, Y. Okuno, and S. Mashiko, Nature (London) 413, 619 (2001); K. Ho, J. Chem. Phys. 117, 11033 (2002); R. M. Tromp and J. B. Jannon, Surf. Rev. Lett. 9, 1565 (2002).

[2] T. Ala-Nissila, R. Ferrando, and S. C. Ying, Adv. Phys. 51, 949 (2002).

[3] J. Wecksesser, J. V. Barth, C. Cai, B. Müller, and K. Kern, Surf. Sci. 431, 168 (1999); J. Weckesser, Ph.D. thesis, Swiss Federal Institute of Technology, Laussane, 2000.

[4] M. Schunack, T. R. Linderoth, F. Rosei, E. Laegsgaard, I. Stensgaard, and F. Besenbacher, Phys. Rev. Lett. 88, 156102 (2002).

[5] G. Ertl and H.-J. Freund, Phys. Today 52(1), 32 (1999).

[6] W. D. Luedtke and U. Landman, Phys. Rev. Lett. 82, 3835 (1999).

[7] P. Jensen, Rev. Mod. Phys. 71, 1695 (1999).

[8] A. C. Levi and M. Kotrla, J. Phys.: Condens. Matter 9, 299 (1997).

[9] K. D. Dobbs and D. J. Doren, J. Chem. Phys. 97, 3722 (1992).

[10] C. R. Henry, Surf. Sci. Rep. 31, 235 (1998).

[11] D. Brockmann and T. Geisel, Phys. Rev. Lett. 90, 170601 (2003).

[12] L. Y. Chen, M. R. Baldan, and S. C. Ying, Phys. Rev. B 54, 8856 (1996).

[13] D. Cowell Senft and G. Ehrlich, Phys. Rev. Lett. 74, 294 (1995); T. R. Linderoth et al., ibid. 78, 4978 (1997); A. P. Graham et al., Phys. Rev. B 56, 10567 (1997); S.-M. Oh et al., Phys. Rev. Lett. 88, 236102 (2002).

[14] J. Ferrón et al., e-print cond-mat/0403565.

[15] M. F. Shlesinger, G. M. Zaslavsky, and J. Klafter, Nature (London) 363, 31 (1993); Applications of Fractional Calculus in Physics, edited by R. Hilfer (World Scientific, Singapore,
2002).

[16] A. Gross, Surf. Sci. Rep. 31, 235 (1998).

[17] K. D. Dobbs and D. J. Doren, J. Chem. Phys. 97, 3722 (1992); R. Ferrando, R. Spadacini, and G. E. Tommei, Phys. Rev. E 48, 2437 (1993); L. Y. Chen, M. R. Baldan, and S. C. Ying, Phys. Rev. B 54, 8856 (1996); J. L Vega, R. Guantes, and S. Miret-Artés, Phys. Chem. Chem. Phys. 4, 4985 (2002); R. Guantes et al., J. Chem. Phys. 119, 2780 (2003); S. J. Mitchell, S. Wang, and P. A. Rikvold, Faraday Discuss. 121, 53 (2002).

[18] J. M. Sancho, A. M. Lacasta, K. Lindenberg, I. M. Sokolov, and A. H. Romero, Phys. Rev. Lett. 92, 250601 (2004).

[19] J. Klafter and G. Zumofen, Phys. Rev. E 49, 4873 (1994).

[20] J. Klafter and G. Zumofen, Physica A 196, 102 (1993).

[21] P. M. Chaikin and T. C. Lubensky, Principles of Condensed Matter Physics (Cambridge University Press, Cambridge, U.K., 1995), Sec. 7.5.

[22] H. A. Makse, S. Havlin, M. Schwartz, and H. E. Stanley, Phys. Rev. E 53, 5445 (1996); E. Koscielny-Bunde, A. Bunde, S. Havlin, H. E. Roman, Y. Goldreich, and H. J. Schellnhuber, Phys. Rev. Lett. 81, 729 (1998).

[23] A. H. Romero and J. M. Sancho, J. Comput. Phys. 156, 1 (1999); A. H. Romero, J. M. Sancho, and K. Lindenberg, Fluct. Noise Lett. 2, L79 (2002).

[24] A. H. Romero and J. M. Sancho, Phys. Rev. E 58, 2833 (1998)

[25] P. Hänggi, P. Talkner, and M. Berkovec, Rev. Mod. Phys. 62, $251(1990)$

[26] J. M. Sancho, A. H. Romero, and K. Lindenberg, J. Chem. Phys. 109, 9888 (1998).

[27] I. S. Gradshteyn and I. M. Ryzhik, Table of Integrals, Series and Products (Academic, New York, 1965). 